vol. 26 - $n^{\circ} 2 \mid 2010$

Numéro ouvert

\title{
De la cohésion de vie du migrant : déplacement migratoire et orientation existentielle
}

The Life-Cohesion of the Migrant. Migration and Existential Orientation

Sobre la cohesión de vida del migrante. Desplazamiento migratorio y orientación existencial

\section{Marc Breviglieri}

\section{(2) OpenEdition}

Journals

\section{Édition électronique}

URL : https://journals.openedition.org/remi/5137

DOI : $10.4000 /$ remi.5137

ISSN : $1777-5418$

Éditeur

Université de Poitiers

\section{Édition imprimée}

Date de publication : 1 septembre 2010

Pagination : $57-76$

ISBN : 978-2-911627-55-2

ISSN : 0765-0752

Référence électronique

Marc Breviglieri, « De la cohésion de vie du migrant : déplacement migratoire et orientation existentielle », Revue européenne des migrations internationales [En ligne], vol. 26 - n² | 2010, mis en ligne le 01 septembre 2013, consulté le 14 avril 2022. URL : http://journals.openedition.org/remi/5137 ; DOI : https://doi.org/10.4000/remi.5137 


\title{
De la cohésion de vie du migrant : déplacement migratoire et orientation existentielle
}

\author{
Marc BREVIGLIERI*
}

\begin{abstract}
"L'ampleur des mouvements migratoires (...) est la preuve que les difficultés économiques sont parfois plus fortes que l'attachement au sol natal. C'est que, dans nos civilisations, il y a une masse humaine relativement déracinée, et qui est prête à se porter là où elle trouvera sa subsistance. Pour arracher cependant les hommes aux pays où ils ont pris leurs habitudes, il faut qu'ils soient entraînés par un courant. Les migrations sont des déplacements collectifs, comparables aux anciennes migrations de peuples : l'émigrant est attiré, puis encadré dans la foule de ceux qui émigrent comme lui » (Halbwachs, 1964 : 221).
\end{abstract}

\begin{abstract}
C'est à l'occasion de sa fameuse réflexion sur la psychologie des classes sociales que Maurice Halbwachs présente sous un tel jour le phénomène migratoire. Dans la première partie de son ouvrage, il s'est déjà interrogé sur la réticence paysanne à « quitter le petit coin où ils (les paysans) ont pris racine, où leur famille vit depuis un temps qui leur paraît indéfini » (1964 : 65). Maurice Halbwachs met en perspective, face à l' " attirance » et l'opportunité que peut représenter la migration, la souffrance d'un arrachement au sol natal, reflétant par là le sort pathétique d'un homme déplacé, dépaysé ou déraciné. En mobilisant la métaphore du « courant », d'autres parleraient de « flux », il ne fait pas que souligner un lien privilégié qu'entretient la mémoire collective avec le déplacement et l'espace, il met aussi en évidence la part de passivité qui accompagne le mouvement migratoire dont la cause semble ici historiquement déterminée. Certes il existe des mobiles d'action qui motivent la migration, mais l'expérience du déplacement migratoire se compose tout aussi bien d'éléments sensibles reçus et vécus sur un mode fondamentalement passif. Il y a des déplacements lestés du poids de la fatalité, des forces impérieuses qui surgissent comme un courant dont la puissance emporte ailleurs, arrache des racines d'existence, pousse à fuir, conduit à lâcher un monde.
\end{abstract}

* Professeur, HETS-Genève et GSPM/EHESS, Rue Prévost-Martin 28, Case postale 80, CH-1211 Genève 4, Suisse ; marc.breviglieri@hesge.ch ; brevig@ehess.fr Je tiens à remercier Matthieu De Castelbajac à qui je dois une relecture attentive et à témoigner de ma gratitude à Fanny Colonna dont l'influence parcourt l'ensemble de ce texte. 


\section{LE DÉPLACEMENT, L'ÉLOIGNEMENT ET LES PATHOLOGIES DE L'EXIL}

\section{Le déplacement et l’impossibilité du retour}

D’une certaine façon, le premier événement subi dans la migration émerge au moment du départ et prend le visage de la séparation des proches, de la perte et d'une certaine rupture avec un monde familier. Et par la suite, ce dernier, malgré la distance, mais non sans rapport avec elle, fait vibrer des points d'adhérence, des lieux d'ancrage, des formes d'attachement sur un fond d'investissement affectif et d'obligations maintenues. C'est notamment le souvenir qui donne à sentir ces vibrations, principalement lorsqu'il appréhende le passé sous la forme d'un itinéraire retraçant la succession des grandes escales de la migration. Par son travail même, et à travers ses modulations, entre mémoire heureuse et mémoire blessée, la mémoire imprime du sens, explique et comprend l'histoire personnelle et collective (Ricœur, 2000). Touchant à l'identité narrative, elle met en rapport un ensemble d'éléments passés à partir desquels se retrace une " continuité intérieure » (Serfaty-Garzon, 2006) et s'atteste un maintien de soi relatif à une cohésion de vie qui s'approprie les ruptures imposées par le déplacement. Et c'est au niveau de cette recherche de cohésion que la mémoire fait se croiser, reflétant dans le mouvement migratoire une dimension inextricablement agie et subie, les parcours personnels aux destins collectifs.

La dimension passive et affectée accompagnant tout déplacement migratoire tient pour beaucoup à la manière dont le temps, nécessairement pris par ce mouvement, interfère avec le franchissement des espaces nouveaux. La migration est aussi du temps qui passe de manière irréversible ; il n'y a pas que le sol natal ou jadis habité qui est laissé derrière soi : un passé aussi s'abandonne. La migration trace un fil qui court dans l'espace et le temps, dessinant une topographie faite d'escales plus ou moins longues, de points de rupture, d'espaces de découverte où, dans chacun des cas, s'est inscrit un avoir été dont le souvenir est «très intrinsèquement associé à des lieux $»^{2}$. Et, de fait, le retour en ces lieux mêmes n'est pas plus envisageable que la rétrogradation vers le passé dans le temps : l'impossibilité de la réversion chronologique englobe en quelque façon l'impossibilité d'un exact repli sur le point de départ, de sorte que tout souvenir enferme la possibilité du regret ou du remords, d'un passé qu'on souhaite faire revivre ou qu'on désire anéantir (Jankélévitch, 1974). Dans son essai sur L'irréversible et la nostalgie, Vladimir Jankélévitch souligne que l'amertume du fait d'avoir-été demeure le cœur du «mal d'exil »; son remède ne peut donc pas être le retour au pays et son avènement reste incontournable : tout migrant sait qu'il ne pourra jamais retrouver le monde quitté qui, lui-même, a été affecté par le temps qui passe (Jankélévitch, 1974 : 290).

2 Paul Ricœur avance que : « la transition de la mémoire corporelle à la mémoire des lieux est assurée par des actes aussi importants que s'orienter, se déplacer, et plus que tout habiter. (...) Ainsi les "choses" souvenues sont-elles intrinsèquement associées à des lieux. Et ce n'est pas par mégarde que nous disons de ce qui est advenu qu'il a eu lieu » (Ricœur, $2000: 49$ ).

REMI 2010 (26) 2 pp. 57-76 
En partant de la seule question de l'immigration, certes la plus sensible dans l'opinion publique et donc la plus brûlante au niveau du débat politique, un ensemble de dimensions propres au phénomène migratoire sont négligées et tenues en réserve. C'est fréquemment le cas des dimensions de l'existence qui, accompagnant les trois moments du partir, du rester et du revenir, qui sommairement dessinent l'arc expérientiel de la migration, sont vécues sur un mode passif. L'appréciation de ce qui est vécu sur un mode passif est particulièrement délicate à envisager depuis la parole du déplacé dans la mesure où celle-ci tend spontanément à réintroduire des contenus d'activité lorsqu'il s'agit de décrire un phénomène qui s'apparente à un déplacement dans l'espace. Ainsi, les analyses portant sur l'immigration stricto sensu interrogent en premier lieu un phénomène actif (l'entrée et l'installation dans un pays d'accueil) rapporté à ses conditions de possibilité. Elles mettent alors l'accent tant sur l'« acteur-migrant » (doté ou non d'un complexe d'acquisitions graduelles et circonstanciées de capacités à l'intégration) que sur l' « acteurrecevant ", souvent collectif, et potentiellement coupable de la difficile intégration du premier (entreprises, administrations ou citadins générant des actes de ségrégation, de déconsidération ou plus gravement encore, de discrimination).

\section{La direction de l'îlot dépressif (A. Sayad)}

C'est en rendant indissociable la condition de l'immigré de celle de l'émigré, « tout immigré restant toujours, sous quelque rapport, un émigré de quelque part (et cela, même dans le cas où cet "immigré" est né dans l'immigration et n'a lui-même émigré de nulle part) », qu'Abdelmalek Sayad ouvre une brèche essentielle dans l'analyse des dimensions existentielles négatives et passivement subies dans les déplacements migratoires. Une ambition qu'il affiche à cet égard est de couvrir un large ensemble d'éléments relatifs au « mal et au mal-être qu'on éprouve dans l'immigration » (Sayad, 1999 : 115). Au premier rang de ces éléments, il place le sentiment de la faute rapportée au départ et à l'absence. Une marge est laissée à l'analyse d'une culpabilité qui ne soit pas entièrement perçue comme pesant sur le pays d'accueil lorsqu'il manque à ses devoirs d'hospitalité et affiche ou ne contient pas une certaine xénophobie. Abdelmalek Sayad montre comment un ressort de la culpabilité du migrant tient au lien qui continue à l'enserrer à ses origines et donc au pays d'émigration. Ce lien nourrit un ensemble de phénomènes perçus, mais non voulus, un pâtir qui expose le migrant à une souffrance dont l'angoisse de la séparation, et son expérience négative, est le révélateur privilégié. Plus précisément, on peut distinguer trois dimensions de souffrance, pour lesquelles on élucidera plus loin quelques connexions souterraines, sous des visages aussi différents que $(i)$ l'impression d'avoir laissé derrière soi le sentiment de la trahison ou du reniement, impression glissant vers la perception du déshonneur, (ii) l' « obsession du retour au passé », penchant vers les expressions pathologiques du désespoir qui bifurque vers la mélancolie ou vers la sinistrose ou 
(iii) le sentiment profond de solitude dérivant vers le mutisme et l'exclusion ${ }^{3}$.

Ces trois dimensions de souffrance, qui semblent affecter avec une intensité toute particulière l'expérience du déplacement migratoire, demeurent, pour ainsi dire, au passif des personnes. Elles sont mises en réserve et tenues dans une dimension de latence, sur une couche existentielle antérieure, mais conditionnelle aux capacités d'adaptation requises dans toute dynamique d'installation. Elles sont celles par quoi vient le découragement à vouloir habiter dans un ailleurs et pourquoi se distend tout ressort tendu vers l'autre (Breviglieri, 2002).

$\mathrm{Au}$ fond, un lien analytique de causalité pourrait faire se recouper ces trois dimensions de souffrance. La dimension de la culpabilité (touchant à la faute de l'absence et aux conséquences imputées à l'abandon des siens), qu'Abdelmalek Sayad préserve à juste titre au rang d'un mal premier, n'est pas sans effet direct sur les pathologies de l'exil (mélancolie ou sinistrose) dès lors que le migrant perçoit son parcours comme la marque d'une fatalité historique et d'un malheur prédestiné. D'un côté, le constat de l'irréversible, ruinant d'une certaine façon la possibilité de la réparation, et de l'autre côté, l'enlisement dans l'amertume de l'avoir-été, isolant sur un îlot dépressif, amplifient les préjudices moraux et psychologiques attachés au sentiment de culpabilité ${ }^{4}$. Mais un autre rapport de causalité pourrait aussi permettre de relier les formes aiguës du désespoir avec les dérives malheureuses de la solitude. En brisant ou en voyant se déliter toute forme de communication avec le monde, le migrant viendrait à compromettre toute possibilité de participation et de reconnaissance sur l'espace public. C'est alors dans un monde désolé, vidé de contacts, appauvri en réseaux qu'il vit son exil ; une vie subie sur le mode de l'exclusion dont un symptôme morbide, dont la gravité culmine aussi sur une échelle de valeur politique, devient le mutisme.

On associe souvent le mutisme frappant l'immigré à son incapacité à prendre part au débat public et à s'introduire dans le monde politique. Ce mutisme est alors associé à l'absence d'une voix participative, représentant la condition d'une action publique, d'une appartenance pleine et de l'instauration d'un monde commun (Breviglieri et StavoDebauge, 2004). Qu'un langage affecté qui ne soit pas celui de la participation continue de sourdre malgré l'existence de ces trois domaines de souffrance, c'est le constat vers lequel conduit la scansion possible d'une tonalité nostalgique dans le monde quotidien du migrant. La nostalgie n'est en effet pas étrangère à certains des maux de l'exil que nous venons d'énumérer car, d'une part elle demeure entièrement imprégnée du pâti de l'expérience migratoire, et que, d'autre part, elle se nourrit de ces maux pour pouvoir

3 Un ensemble de maux qui, pour Abdelmalek Sayad, représente un « coût social » pour l'émigration. Il faut, avance-t-il, pour que ce coût soit supporté, que le migrant soit capable de trouver un sens à son existence suffisamment profond pour en contrebalancer les effets négatifs. La vulnérabilité des immigrés, telle qu'il la met à jour, repose alors essentiellement sur leur « incapacité (sociale) à se situer dans une perspective qui donne sens à leur existence », et sur le fait que ce sens ait surinvesti, non sans perversité, la sphère du travail qui a partie liée avec la migration algérienne en particulier. Et cela survient tout à la fois au principe et au fondement de la «faute originelle » qui naît de l'absence (Sayad, 1999).

4 La mélancolie renvoie à la clôture de l'îlot dépressif et l'« improductivité existentielle » tandis que la sinistrose suppose un mécanisme d'amplification du préjudice subi (Binswanger, 1987).

REMI 2010 (26) 2 pp. 57-76 
resurgir dans son propre registre expressif. Il reste, comme le suggère Paul Ricœur, que la nostalgie convoque un "soupçon érigé bien souvent en reproche " ${ }^{5}$. Placée sous un tel regard suspicieux, la nostalgie rendrait tangible une expression résiduelle, dénuée de substance politique, et non pas une parole résistante. Elle demeurerait incapable de convertir un malaise ou une plainte en accusation imputant un tort à un agent responsable, elle rassemblerait autour d'elle, non pas dans l'opposition ou l'affrontement qui restent des caractères agonistiques irréductibles au plan du politique, mais par contagion sensible, diffusant de proche en proche une tonalité affective touchant au plus intime de l'être.

La nostalgie contribue plus empiriquement à frapper de discrédit le lieu où je me tiens au regard de celui d'où je viens (Giraud, 2009). Elle tend à faire de l'expérience contingente, non pas un moment critique de l'aventure humaine, mais une dérive de la vigilance qui perd ses armes devant les réalités de l'exil. Une vigilance désarmée face au temps présent, notamment car la nostalgie s'arrime à une dynamique émotionnelle où s'entremêlent la mémoire et l'imagination. Et c'est à la lueur du regret que survient du passé et s'impose au présent un lieu partiellement imaginaire, paré d'un prestige tout particulier malgré les brisures de l'absence et les plaies de l'abandon. C'est alors, bien sûr, une difficile économie du regret, faite de compensations et de volonté d'oubli (Villanova, 1999), que s'évite l'écueil de l'îlot dépressif et que s'envisage un aspect essentiel de la fragilité identitaire du migrant. Une économie du regret dont la tâche essentielle demeure de maintenir ce dernier dans une cohésion et une continuité du soi qui préexiste à toute capacité d'adaptation et d'intégration.

\section{LA NOSTALGIE ET LA SUTURE AFFECTIVE AU LIEU D'ORIGINE}

\section{Le retour comme point visé à l'horizon de la cohérence d'une vie (nostalgies)}

L'histoire du concept de nostalgie réserve quelques enseignements importants sur les blessures de la migration, et notamment au fil de ses déplacements sémantiques. Ce néologisme a été formé par le médecin alsacien Johannes Hofer à la fin du XVIIe siècle (Hofer, 1934 et 1992). Il désignait alors une maladie causée par le mal du pays et qui associait un état dépressif à de graves perturbations physiologiques. Comme le typhus, la nostalgie était placée sous la catégorie générique des maux contagieux, et elle était perçue comme une cause de la folie, de certains suicides et de certains cas de désertion de l'armée dans la mesure où elle toucha pour commencer des montagnards suisses, mercenaires engagés dans des armées et se retrouvant au combat dans des plaines (Bolzinger, 1999). Les thèses ont vite divergé concernant l'origine même de la nostalgie : était-ce, pour ces montagnards, une question de pression atmosphérique différente, l'air de la patrie qui venait à faire défaut ou bien la souffrance de n'avoir jamais quitté auparavant la maison familiale et l'impossibilité d'oublier les soins dont les entouraient leurs mères ? Il fut

5 Ce passage est tiré d'un texte consacré à la place centrale du politique dans l'œuvre d'Hannah Arendt (Ricœur, $1991: 16$ ). 
question dans certains corps d'armée d'interdire à ces mercenaires de chanter ou de siffler des airs musicaux rappelant la terre d'origine, car ils semblaient accroitre considérablement le sentiment nostalgique et ses pathologies conséquentes. Deux éléments relatifs au déplacement migratoire sont impliqués dans cette première région de sens touchant à la nostalgie : d'une part, elle reflète les conséquences d'un déracinement et suppose que le sujet affecté préserve et valorise un ensemble d'impressions issues des lieux familiers où se sont inscrites des expériences primordiales ; d'autre part, la musique et ses mélodies, davantage que la simple parole, sont des facteurs qui la déclenchent et la diffusent par effet de contagion. Ainsi, ce qu'elle manifeste au plan affectif, ses formes affectives d'énonciation, semble plus important que ce qu'elle pourrait énoncer au plan verbal.

Le concept de nostalgie va ensuite être victime d'un relatif oubli dû à la manière dont va s'affirmer la recherche médicale aux XVIIIe et XIXe siècles où prévalent, notamment parce que se développe massivement la pratique de l'autopsie, les causes anatomiques de la maladie aux dépens des explications psychologiques. C'est par la médecine légale que la nostalgie va refaire une apparition remarquable reflétant un mouvement de réémergence de la psychologie dans le champ médical de l'époque. Au début du XXe siècle, Karl Jaspers réalise sa thèse intitulée Nostalgie et criminalité. Il s'y trouve une tentative pour comprendre certains crimes inexplicables en rapport à la douleur morale du déracinement et à l'obsession de retourner dans les lieux de l'enfance qu'il a fallu quitter (Bolzinger, 1999 : 260). Ces crimes sont généralement commis par de jeunes adolescentes arrachées à leurs familles et placées comme bonnes d'enfants ou filles de ferme. Elles assassinent dans un moment de délire les enfants de bas âge dont elles avaient la garde ou bien brûlent la demeure ou la ferme où elles travaillaient et, meurtrières ou incendiaires, elles tentent de se réfugier dans la maison de leurs propres parents. La potentialité pathogène de la puberté, les dérives impulsives de la conduite adolescente, la force irrépressible produite par un " paroxysme de nostalgie » ont alors eu tendance à orienter le jugement de ces crimes en faveur de l'irresponsabilité pénale (Bolzinger, 1999). La jurisprudence s'est alors retrouvée contrainte puis disposée à évaluer la trajectoire biographique du criminel et non plus simplement ses dispositions psychiques au moment de l'action. Les jeunes filles coupables ne semblent manifester au moment du crime ni haine ni désir de vengeance, mais plutôt des troubles de l'angoisse à partir desquels ont pu s'envisager des dispositions psychiques expliquant une conduite largement réactive et non intentionnelle.

C'est au niveau d'une précompréhension du phénomène migratoire vécu sur un mode passif que nous place en quelque sorte l'usage juridique de ces analyses sur l'expérience pathologique de la nostalgie. En se penchant sur des crimes jugés « inexplicables » et en tentant l'explication de l'irresponsabilité pénale sur la base de l'apparition de conduites impulsives et irréfléchies, l'expertise médicolégale délimite un syndrome subjectif relatif à la séparation physique qui induit le désarroi de la perte et la tension vive vers un retour aux lieux familiers de l'origine. Ce même type de syndrome conduit une psychologue traitant des patients maghrébins déracinés dans l'exil à extraire de son raisonnement un socle d'hypothèses renvoyant aux conditions de la migration : " la distance réelle, géographique, joue un rôle qui n'est pas négligeable, (...) la terre natale offre une sorte de localisation pour le désir, (...) avec un exilé on découvre l'importance de la capacité à éprouver de la nostalgie » (Ayouch Boda, 1999). L'obsession d'un retour, qu'il soit refusé

REMI 2010 (26) 2 pp. 57-76 
ou promis, illusion rassurante ou gouffre d'inquiétude, interroge le plan fondamental de la cohésion biographique du migrant et convoque la question d'une transmission primordiale permettant d'assumer l'empreinte laissée par des éléments archaïques propres au monde familier de l'origine et, en particulier, de l'enfance.

Dans son trajet historique et sémantique, la nostalgie a aussi côtoyé de près l'idéologie du romantisme allemand. C'est à cette condition que son assimilation à une pathologie a pu être relativisée, et que le terme a été approprié par des esprits cultivés qui à la fois regrettent les charmes perdus d'un passé glorieux et tentent l'impossible « reconquête de l'unicité perdue $»^{6}$. Cette inclination romantique à refluer vers un passé idéalisé fait vibrer la grandeur d'un attachement. Ce reflux nostalgique est l'inverse d'une rétraction, il tente d'assumer un passé et sa primordialité au bénéfice de la cohésion identitaire du sujet. Cette cohésion voudrait témoigner d'une capacité à endurer et assumer toute épreuve de séparation subie au fil de l'histoire personnelle et collective. La nostalgie reflète cette inclination au reflux vers le passé, mais elle dramatise l'épreuve de la séparation en associant la terre d'origine, qu'il a fallu quitter, au rêve d'une symbiose nourricière et heureuse et en faisant du souhait de sa reconquête un horizon inaccessible. Et si ce travail de reconquête a pu nourrir les génies musiciens ou littéraires de l'Allemagne romantique, il conduira aussi l'Allemagne moderne vers une pente réactionnaire et vers un désir de totalité et de monde commun unitaire (Rauchs, 1999). Il y a donc, à travers la fragile cohésion identitaire que permet la nostalgie, non seulement un effort remarquable que produit le migrant pour déplacer son lieu d'origine, institué par l'exil même, là où il va ; mais il y a aussi une pente rétrograde, une posture passéiste qui tend à cristalliser ce lieu et à lui donner une forme close et définitive. La cohésion se consolide alors, mais dans une quasi-virtualité problématique car elle ne s'opère qu'à la défaveur de toute rencontre véritable, de toute possibilité de décentrement de perspective, et dans l'impossibilité de fonder un monde commun dans un ailleurs où la médiation de l'autre est requise.

\section{Cristallisations et idéalisations dans l'absence et la distance (des maisons habitées au foyer du nationalisme)}

La nostalgie appelle toujours davantage que la perte de l'avoir-été, elle puise aussi, et c'est pour cela qu'elle laisse un goût doux et amer à la fois, dans la mémoire d'une perception de plénitude liée au sentiment serein d'habiter. Un sentiment qui continue de rayonner, malgré l'éloignement, comme une illusion rassurante. Cette dimension de l'habiter reste en effet l'objet même d'une cristallisation, ce que cherche à embellir et à parer de prestige la nostalgie. Habiter suppose un certain ancrage phénoménal du corps, nourri par une matrice d'expériences familières, un foyer d'émotions sécurisantes procurées dans l'intimité du chez soi, une suture affective qui tient chacun comme attaché aux proches par un lien non-arbitraire (Breviglieri, 2002). À l'horizon de cet ancrage, l'attachement au monde habité reflète certains bienfaits tenant à une constellation d'ha-

6 Paul Rauchs, dans la même livraison que les articles précédents, revient sur le credo de l'Allemagne romantique du 19e et souligne que « morcelée en une multitude de petits États épars, (elle) se languit de la grandeur passée du "Saint Empire Romain de Nation Allemande" ", elle quête une unité qui « s'applique à la politique, bien sûr, mais aussi à une ancienne religion catholique non encore souillée par le schisme luthérien » (Rauchs, 1999 : 286). 
bitudes partagées et à des commodités trouvées dans les murs de l'entre soi. En un sens, ces bienfaits touchent aux dimensions les plus tangibles et immédiates de l'accueil illimité réservé au proche. C'est pourquoi, le sentiment d'habiter se compose d'une intuition tout à la fois éthique et esthétique. La vieille maison de famille, les rivages lointains du pays d'origine, le visage des " anciens » restés là-bas, ne sont pas remarquables au sens des canons esthétiques de l'architecture, du paysage ou de la beauté physique, mais bien dans leur capacité à sembler préserver et soutenir la promesse d'un accueil heureux.

Mais selon quel mécanisme s'annonce, s'énonce et résonne la nostalgie ? Son mode de donation, pour lequel on sait déjà que le contenu exprimé compte moins que sa forme d'énonciation, s'effectue toujours dans la préoccupation d'une séparation et dans la tension due à l'idée de retour. Dans la voix qui contient une note de nostalgie, l'affect de la séparation se décharge et la parole le réoriente en filant une intrigue relative à la possibilité même du retour. Le retour ne s'évoque généralement pas comme un plan raisonnable tracé dans l'avenir, mais par ce qu'on peut saisir du tableau contrasté que dépeint la nostalgie : le « là-bas » convoité s'oppose à l'ici et au maintenant comme le plein s'oppose au vide, comme l'intensité du sentiment d'habiter s'oppose au rien qui demeure à jamais inhabitable. L'opposition polaire produit un effet d'aspiration, le retour est alors perçu comme un appel pressant.

Il reste que la nostalgie fait venir, dans la préoccupation de la séparation, l'objet d'un regret. Or, le regret a deux faces très voisines. S'il n'est pas regret du passé intime, il est regret d'une chose idéale que l'on n'a pas eue, et qu'on peut souhaiter voir apparaître. C'est à partir de là, et dans un mouvement d'idéalisation, que se dépasse l'horizon convoqué par la simple évocation d'un vécu intime. La singularité du souvenir y est transcendée et la nostalgie peut s'ouvrir potentiellement à des échelles qui enveloppent des communautés plus larges que le simple lieu que le corps a habité dans le vis-à-vis des proches. Ce que fige alors la matrice de l'idéalisation, ce sont des images stéréotypées qui étendent les lieux familiers en terre d'origine, qui grandissent la sécurité ontologique procurant le sentiment serein de pouvoir habiter en fierté d'appartenir et en volonté de manifester une appartenance.

Même lorsqu'elle se déploie jusqu'au mal du pays, et dans ce qui touche à la patrie et à la communauté d'origine, quelque chose de l'habiter résonne dans la nostalgie. Mais quelles sont les voies de passage qu'emprunte la nostalgie pour tendre ainsi vers la mise en valeur de la communauté d'origine ? Ce que la nostalgie engage en premier lieu, dans le geste énonciatif qui nous intéresse ici, c'est la métaphorisation des propriétés de la communauté et de la collectivité à partir des qualités élémentaires de la maison. Pour cela, la nostalgie puise dans des concepts permettant des chevauchements notionnels. Parmi ces concepts capables de caractériser certains traits de la maison comme ceux du pays, les plus cruciaux restent sans aucun doute ceux de la convivialité, de la sécurité et de l'authenticité dans leur rapport étroit à la tradition. Chacun de ces traits donne accès à une certaine réalité de la frontière qui tiendrait à distance l'hostilité et l'inauthenticité du différent. Avec la nostalgie, la communauté tend à prendre sens à partir de l'image archétypale de la maison, comme enceinte fondatrice et lieu de la natalité, comme pourvue d'un mur qui protège et sépare un intérieur d'un extérieur, comme procurant un foyer dont le centre est chaud comme la convivialité et contraste avec le froid de l'extérieur où siège l'indiffé- 
rence et la solitude. On aperçoit alors que ces concepts manifestent entre eux un évident « air de famille » en cela qu'ils reflètent tous des formes convergentes de politiques, et particulièrement celles qui sont à même de valoriser les dimensions sécuritaires, protectionnistes et patrimoniales de la nation.

\section{LIGNES DE CONTINUITÉ OU TOURNANTS DE VIE ?}

\section{L'orientation des engagements passés}

Plusieurs éléments ont été avancés au terme de cette première série de réflexions. On est parti de l'idée que le migrant, peu importe les mobiles de son déplacement (migrant de travail, regroupé familial, déplacé, exilé ou réfugié), vit cette expérience avec une part de passivité et d'involontaire. Cette expérience se rapporte souvent à de profonds bouleversements personnels ou collectifs et à l'émergence de véritables enjeux d'orientation existentielle aux trois stades biographiques du départ, de l'installation et du retour possible. Parce que ces orientations correspondent pour le migrant à des tournants à savoir négocier dans l'existence, les phénomènes de la migration et de l'exil imposent de considérer, en contrepartie de ce savoir, une gestion de la complexité et un effort pour répondre à un besoin primitif de cohésion malgré les déplacements entrepris et subis. Tout déplacement prenant l'envergure d'une migration rappelle le lien de constitution réciproque entre une intériorité psychique et un espace parcouru (Binswanger, 1998), et il place au cœur de l'espace existentiel le problème de l'orientation depuis lequel migrer pose les questions du vers-où et du pour-quoi de l'existence. Il y a en jeu une capacité à gérer dans le déplacement le bien fondamental à l'échelle de la personne que représente la cohésion de soi. Cet appel aux capacités de négociation et de gestion consacre au plan analytique une extension du domaine de réflexion de l'économie, qu'on rapporte ici, dans un premier temps, à l'économie de la « maison intérieure » où s'organisent les orientations existentielles élémentaires ${ }^{7}$.

On a déjà souligné, en un sens, l'importance, dans le déplacement, du monde laissé derrière soi. C'est sous l'angle d'un désir de communauté qui est aussi nostalgie de l'unité originelle, la perte d'un monde commun renvoyant indissociablement à une perte de contact avec sa propre histoire de vie, que la question a été ouverte. Le sentiment de perte d'une communauté d'appartenance peut donner un sens négatif à l'existence du migrant, il peut aussi l'orienter vers une quête chimérique animée par l'avoir été et tournée vers le passé idéalisé. L'investissement émotionnel dirigé vers le monde-derrière-soi met en péril le migrant s'il n'est pas subordonné à une économie du regret capable de tempérer

7 L'expression " maison intérieure » renvoie ici à Frey, 2004. On verra par la suite que cette économie, qui permet de tracer des chemins de cohérence pour la personne déplacée, recouvre aussi les dimensions de la morale et de l'échange, de l'obligation et du symbolique, dimensions à partir desquelles le bien mis en jeu apparaît plus manifestement comme un bien commun, c'està-dire un bien rapporté à l'échelle des communautés d'accueil et d'origine. Un certain nombre d'éléments présentés dans cet article ont été discutés dans le séminaire «Extension de l'économie politique : fascination, oppression, dépression » que j'ai coordonné avec Nicolas Auray et Laurent Thévenot à l'École des Hautes Études en Sciences Sociales en 2008-2009. 
la force de cette quête et de ce sentiment, et dont une fonction remarquable est de permettre de supporter des blessures que le temps n'est pas capable d'effacer. Cette économie du regret s'est imposée à la réflexion comme le premier élément pour comprendre la fragilité identitaire et le difficile maintien d'une cohésion de vie mise à l'épreuve d'un déplacement migratoire (voir supra). La nostalgie est d'une certaine manière un produit de cette économie à partir de laquelle le migrant gère tant une douleur sensible dans l'éloignement, qu'une grandeur dans l'attachement préservé au lieu d'origine. Mais on pourrait délimiter une économie dont l'orientation est tendanciellement inverse : au lieu de subir pleinement ces phénomènes par la médiation de la nostalgie, le migrant incline à s'en déprendre. Autrement dit, le déplacement migratoire recèle schématiquement deux tendances prêtes à se dissocier : l'une qui cherche à déplacer intégralement le lieu d'origine, à l' « emmener » avec soi, et la nostalgie relève de cette tendance, et l'autre qui ignore ou abdique devant un tel effort, semblant fuir ce qui touche au passé, être fasciné par le renouveau, répondre d'un besoin de renaître ou de la tentation de l'oubli.

En portant l'attention sur ce qui est subi passivement dans le déplacement migratoire, on est toutefois conduit à relativiser profondément la conception anthropologique d'un migrant qui serait animé par ses seules ressources de mobilité le propulsant dans un espace de choix de destinations. Les images du « réseau migratoire » ou de l' « espace de circulation » rendent d'ailleurs assez mal compte de la réalité de l'existence des migrants dont il est ici question et ce, sans qu'il faille remettre en cause l'existence d'efforts de construction d'espaces de circulation transnationaux ou l'importante activité de tissage de réseaux transnationaux qui se déforment selon l'émergence d'opportunités économico-juridiques d'installation (Potot, 2007). Le choix de la notion de déplacement entend précisément faire venir les charges affectives et morales qui accompagnent le phénomène migratoire et dont on ne se dégage pas simplement sous l'effet de la volonté de les oublier. Le déplacement suppose en un sens d'établir et de préserver un sol de concordance entre des mondes éloignés. Un tel effort ne s'impose pas uniquement pour établir une connexion entre deux mondes étrangers, mais aussi pour des raisons de continuité dans les parcours migratoires, eux-mêmes situés dans des enjeux plus vastes de cohésion biographique dans la vie des migrants. La notion de déplacement permet donc de tenir en vue la persistance d'un ensemble d'attentes et d'obligations qui se rapportent à la communauté d'origine. Le lien alors concerné peut bien toucher des sphères de vie ou des domaines d'activité aussi variés que l'économique, le politique, l'administratif, les relations de voisinage, les liens familiaux, amicaux ou amoureux. Ce lien, maintenu malgré la distance, renvoie à des formes variées d'engagements qui, en quelque sorte, font survivre la communauté d'origine (Thévenot, 2006) : les alliances et les pactes, dûment scellés avant le départ ; l'attachement ou la haine personnelle, profondément enracinés et redoublés dans la distance et l'éloignement; l'obligation de soutenir une famille et le devoir de porter haut l'honneur d'un clan, etc. ${ }^{8}$.

8 Dans un monde où les courants de migration s'accélèrent, il n'est pas pertinent de considérer une seule et unique communauté d'origine. Il faut, pour pouvoir conserver ce terme dans l'analyse, dissocier une acception de la communauté d'origine entendue comme celle où l'on naît d'une acception entendue comme celle d'où l'on vient. On retiendra cette seconde acception qui nous permet de considérer, lorsque les parcours de migration sont complexes, une possible démultiplication de l'origine des engagements que persiste à entendre le migrant dans ses déplacements.

REMI 2010 (26) 2 pp. 57-76 


\section{La « tentation de brûler les ponts derrière soi » (G. Simmel)}

Toutefois, et bien que ces engagements aient le pouvoir de sourdre à distance, le déplacement migratoire peut présupposer ou faire venir de lui-même une matrice affective se rapprochant du désir de mobilité. Ce désir peut lui-même faire fonds sur une tentation de tourner la page, de s'évanouir d'un monde pour que le surgissement corrélatif dans un ailleurs soit d'autant plus sensible. Il serait réducteur d'ignorer ce principe animateur sous prétexte que des points d'adhérence au lieu d'origine généralement subsistent. Le considérer dans son importance c'est admettre que la mobilité du migrant lui offre non seulement la possibilité de changer de cap, de modifier ses points de vue ou d'ouvrir des perspectives inédites, mais qu'elle peut aussi avoir partie liée avec la rupture des engagements le tenant lié à sa communauté d'origine. En réalité, tout déplacement migratoire a la potentialité de réorienter l'existence et de marquer un tournant de vie radical et définitif. Migrer suppose devoir qualifier un nouvel espace autour de soi et mettre en chantier de nouvelles bases concrètes définissant un ici d'où se livre le nouveau monde. C'est alors qu'on peut découvrir, dirait le géographe phénoménologue Eric Dardel, une " réalité géographique » toute différente, dotée d'une autre " intensité existentielle » et convoquant, au fond, une " nouvelle condition terrestre » (Dardel, 1990). La tonalité du déplacement contient, au regard de cette intensité existentielle, le " goût de l'aventure », c'est-à-dire de l'expérience d'un événement pouvant être détaché du reste de la vie et l'entacher d'une certaine discontinuité biographique. L'aventure, en effet, suppose de « s'isoler en quelque sorte de l'ensemble de la vie », de pouvoir se montrer " indépendant d'un avant et d'un après »; et c'est pourquoi Georg Simmel dit qu'elle « décline la participation à la continuité de la vie », qu'elle permet à l'aventurier de « brûler les ponts derrière lui » (Simmel, $2002:$ 71,72 et 78).

Le problème de la cohésion de vie refait surface. Il fait inlassablement se rejouer les deux courants d'orientation, les deux inclinations tendancielles déjà mentionnées plus haut. Mais on perçoit mieux désormais comment leur dissociation vient interroger différemment le problème en question : dans le premier cas, illustré par la nostalgie, cette cohésion est comme un horizon fuyant car le migrant se tient rivé à un passé qui projette vers lui une dette infinie, ce qui fait que l'unité tant désirée avec la communauté d'origine toujours s'échappe ; dans le second cas, illustré par l'aventure que Georg Simmel a dépeinte comme « un processus de la vie qui s'accélère jusqu'au point d'effacer passé et futur » (Simmel, 2002 : 83), la cohésion est menacée tant par la valorisation de l'indifférence qui néglige le soutien cohésif apporté par l'expérience d'autrui, que par la tentation de la dispersion quotidienne qui fait perdre de vue les convictions personnelles profondes et, comme le note Simmel, qui livre l'individu au hasard et aux caprices de la chance qui peuvent bien rompre le fil de l'unité de la vie (Simmel, 2002 : 74).

On voit néanmoins tout l'intérêt pour l'analyse de se pencher sur le conflit entre ces deux courants d'orientation existentielle, en laissant a priori indécidable sa résolution. Ils font surgir des polarités qui s'opposent et s'attirent à la fois. C'est d'ailleurs un noyau d'intelligibilité qui se dégage clairement des travaux de l'École de Chicago sur la socialité métropolitaine. L'isolement des communautés tournées vers leurs habitudes et leurs valeurs sentimentales, attitude qui représente aux yeux de Robert Park les « formes élémentaires du conservatisme », est mis en tension avec la «fusion des cultures régio- 
nales et tribales » qu'introduit une civilisation urbaine où « l'ingéniosité » du citadin prévaut sur son « traditionalisme » (Park, 1984 : 100 et 168). Le mélange intégral de populations migrantes implique chez chacun une souplesse de confrontation capable de briser des attachements et des clôtures pour pouvoir cohabiter avec la différence de l'autre? Mais cela n'empêche pas que les " situations cosmopolites » oscillent fondamentalement entre ces deux pôles, y compris dans les contextes actuels d'échanges économiques mondialisés où persiste le souci d'entretenir des spécificités rapportées à l'origine, de tracer des «frontières ethniques », fussent-elles poreuses, ou d'assumer une « filiation historique » tout en pouvant « rejeter la prééminence de clôtures » culturelles (Hily et Rinaudo, 2003 : 55). Ainsi, l'emprise des « contenus de vie » du passé et la tentation d'adhérer aux processus de la vie rivés au présent peuvent s'affecter réciproquement dans une dialectique que connaissent bien les migrants et dont l'indice majeur reste le problème du dédoublement identitaire et de la sensation d'instabilité entre deux cultures que traduit le sentiment ambigu, lorsqu'il se trouve logé entre peur et attente, de devoir « rentrer chez soi » (Dufoix, 1998).

\section{Le troublant sentiment de devoir rentrer}

Avec la question si délicate du retour au pays d'origine, on touche à l'aspect le plus épineux des problèmes éthiques et politiques concernant le migrant dont on peut dire, avec Joan Stavo-Debauge, qu'ils culminent nécessairement au niveau fondamental que posent les questions de la juste appartenance et de la bonne hospitalité (Stavo-Debauge, 2009). Peut-on en effet, et sous quelle condition, continuer d'avancer qu'une communauté politique est hospitalière dès lors qu'elle souhaite offrir aux immigrés la possibilité d'un retour sur leurs lieux d'origine? Le mouvement fondamental de l'hospitalité n'est-il pas un geste qui demande de rester, et, sur le fond, peut-on borner ou limiter l'hospitalité sans la déprécier ou la dégrader? La question, pourtant placée sous le signe de l'équivocité, ne semble pas creuse ni vaine après que l'attention a été portée sur la persistance de certaines formes d'attachement, sur le fait que le migrant reste arrimé à quelques ports d'attache situés sur sa terre d'origine et dans différents lieux où s'est posé son parcours migratoire, et dès lors qu'on soutient que « la migration sous toutes ses formes (...) est hantée par l'idée du retour dont l'ultime question se trouve être celle du lieu où l'on doit enterrer celui qui n'est pas mort dans sa patrie » (Dufoix, $1998: 79$ ).

Il faut ainsi accorder une considération au fait que les formes variées d'engagement qui accompagnent le migrant au fil de ses déplacements migratoires, et qui résonnent depuis le lieu même de l'émigration, exercent des tensions qui vont jusqu'à fonder chez lui le sens d'une obligation, plus ou moins puissante, à devoir rentrer. Même si des études

9 L'École de l'écologie urbaine de Chicago a ainsi montré la corrélation entre le développement de la grande ville, fondé sur les flux migratoires, et l'avènement d'une morale rendant possible la coexistence des peuples migrants. Cette morale n'est pas sans lien avec un mouvement de sécularisation, où s'efface la limite entre le sacré et le profane, et avec la prédominance de rapports interhumains fondés sur une indifférence polie. Joan Stavo-Debauge, y voyant le déploiement d'un véritable "modèle de l'hospitalité », particulièrement efficiente dans des communautés à vocation internationale et multiculturaliste, montre comment cette école plonge ses racines tant dans un pragmatisme philosophique que dans un libéralisme politique classique (Stavo-Debauge, 2009). 
ont montré que ce sens passe par des états variables, que le choix ou le projet du retour fait souvent place au fil du temps à un « mythe du retour » où la possibilité même du retour s'épuise, «l'idée de retour n'est jamais totalement abandonnée » et elle reste toujours connotée par la nécessité de « bien revenir» (Laffort, 2005 : 110). Qu'il s'exerce sur une modalité opportune ou impérative (lorsque le migrant s'échappe brusquement pour un retour dicté par un événement particulier - politique, cérémoniel, affectif -), régulière et automatique (correspondant pour certains à la période des vacances scolaires), ou comme l'aboutissement d'un projet de longue date, le retour s'inscrit dans une forme d'économie.

On avait vu que, dans l'exil, le déracinement appelait une économie du regret capable de produire un apaisement soulageant les déchirements de l'être intérieur du migrant. Il est temps d'élargir cette notion d'économie afin d'appréhender plus justement ce qui fonde le sentiment ambigu de devoir rentrer. Au total, on peut même dire que l'on a affaire à trois économies différentes dont il faut d'emblée souligner l'interdépendance : une économie du regret, donc, à partir de laquelle s'appréhende la condition de l'exil sur un plan pathique ; une économie de la dette qui élève le regret sur un plan moral qui appelle à se rendre responsable des conséquences de la séparation et d'un avenir qui engage malgré la distance ; une économie matérielle et symbolique qui inscrit l'acquittement de la dette dans une logique d'affirmation d'une réussite sociale qui, au-delà de ce qui est dû, réclame l'affichage à tout prix d'une réussite. À chacune de ces économies correspondent des formes de faillite ouvrant sur différents malaises, mal-être ou peines endurées dans l'exil (tour à tour : mélancolie et sentiment du manque, culpabilité et remords, frustration et humiliation). Cette qualification des peines vient à son tour éclairer les formes de temporalité impliquées dans chaque économie : au temps de l'économie du regret, dont la bonne marche repose sur une perception évolutive et raisonnable d'un passé fondateur, s'oppose la conception circulaire du temps impliquée par l'économie de la dette qui diffère à son tour de l'idée d'un temps devant porter une trajectoire ascendante, une progression et un enrichissement dans l'économie symbolique de $1^{\prime}$ 'exil ${ }^{10}$. C'est dans l'accumulation et la confusion de ces différents registres temporels d'implications, que les peines et souffrances endurées dans les déplacements migratoires peuvent être tenues pour variables, complexes et souvent profondes.

\section{LES TROIS ÉCONOMIES IMPLIQUÉES PAR LE DÉPLACEMENT MIGRATOIRE}

\section{Partir/rester/revenir (M. Mauss)}

On doit à Marcel Mauss une tentative fameuse pour penser le dépassement d'une économie fondée sur la forme marchande de l'échange, et d'avoir incarné celui-ci par la figure du don. L'effort de Marcel Mauss repose en partie sur l'édification d'une dynamique d'échange reposant sur le lien de trois types d'obligation (donner/recevoir/rendre) assurant la circularité du don et faisant du même coup société étant donné que chacun de ces actes a vocation, non seulement de consolider des interdépendances, mais aussi de prendre un

10 À propos de la temporalité de la dette, voir Sarthou-Lajus, 1997 : 71-91. 
caractère cérémoniel, de produire un « transport de matière symbolique » et donc de se manifester publiquement (Karsenti, 1994). En revenant sur la schématisation qui semble dessiner l'arc expérientiel de la migration, elle aussi triadique (partir/rester/revenir), il paraît envisageable d'approfondir la perspective maussienne tout en lui donnant une ambition d'une tout autre envergure. Non seulement cette seconde triade introduit très directement la question du rapport à l'espace, et l'on s'est efforcé de regarder ce que produit en propre le fait de quitter un lieu, de s'éloigner d'un environnement familier et d'être tenu à distance d'un monde où l'on aperçoit sa propre origine, mais elle modifie aussi les termes d'appréhension de la question de l'échange. Puisqu'elle est initiée par un mouvement de départ et de retrait, l'analyse s'ouvre d'abord à la question de l'abandon plutôt qu'à celle du don ${ }^{11}$. Enfin, en orientant son analyse vers la compréhension d'une grandeur publique dont la dynamique repose sur la prodigalité et l'honneur (Mauss, 1993 : 177), Marcel Mauss rend imperceptible des échelles d'intensité de valeur qui ne sont pas publiques et tiennent notamment aux relations maintenues ou distendues entre proches (Thévenot, 2006).

Nous l'avons vu, c'est sur le troisième terme de la seconde triade, le retour, que semble reposer la principale intrigue affective et narrative du parcours de la migration. Une part importante des conversations ordinaires du migrant touchant à son lieu d'origine, et ce d'autant plus qu'elles croisent d'autres points de vue de migrants, provoque une attraction spontanée (et mutuelle) pour le retour : " pour combien de temps es-tu là ? Comment vont tes proches, te manquent-ils ? Iras-tu leur rendre visite cet été ? », etc. ${ }^{12}$. On ne peut pas présager exactement quand prendra fin le périple du migrant, quand soufflera assez fort le désir du retour, quand la nostalgie pliera la volonté de rentrer pour que s'initie l'acte de revenir. Il est difficile aussi de bien pouvoir saisir ce qui émerge à la réalité lorsque se ferme la possibilité même du retour, combien se tend alors l'impatience de revenir, sur quel support est alors dévié ce désir refoulé. La nostalgie n'a-t-elle pas alors ce rôle ambigu de tenir éveillé le manque tout en offrant une modalité imaginaire de retour permise par la tension rêveuse qui se dégage des souvenirs poignants?

Comme Paul Ricœur l'a remarqué dans son essai abordant le statut du terme « reconnaissance », Marcel Mauss a déjà, dans son Essai sur le don, fait porter toute l'intrigue de la triade donner/recevoir/rendre sur le troisième terme : qu'est-ce qui oblige à donner en retour (Ricœur, 2004) ? On connaît la solution que Mauss propose, à savoir que la force qui oblige à rendre réside dans la chose même, à la façon d'une énergie magique capable de rapporter le bien échangé au lieu de sa naissance (Ricœur, 2004 : 342). Une évidente parenté entre les deux triades repose sur l'analogie du « faire retour» et sur le fait même que les retours pour le migrant, même non définitifs, restent l'occasion d'ouvrir un moment festif où des offrandes sont habituellement faites à ceux qui sont

11 Dans le modèle maussien, c'est bien sous l'aspect positif du don, et non comme abandon de la chose donnée, sa contrepartie négative, que le phénomène étudié est décrit. Dans la migration, le départ certes emporte l'espoir d'une réussite. Mais celle-ci n'est pointée que sur un horizon nécessairement postérieur à l'ébranlement du cercle des proches et à la fissure du monde habité que la séparation provoque brutalement. De sorte que, au moment de l'adieu, l'espoir de réussite peut bien être recouvert par le seul souci de pouvoir rejoindre ou reconquérir des proches dans l'exil.

12 Sur l'émergence du lien à l'origine dans les conversations ordinaires, voir Breviglieri, 2001.

REMI 2010 (26) 2 pp. 57-76 
restés. Mais l'on pressent aussi tout l'écart qui sépare les deux phénomènes entendus par ce même mouvement de retour : ce sont des mouvements différents en configuration et en substance, et cela se perçoit déjà à partir de la sécheresse émotionnelle qui accompagne la triade maussienne et contraste fortement avec la puissance émotive des retrouvailles que peut connaître le migrant. Les retours vers le lieu d'origine s'inscrivent d'ailleurs sur un socle émotionnel riche et instable. Il peut s'y célébrer une fidélité préservée, s'y jouer une fierté de l'appartenance, une volupté à combler soudain un manque, mais aussi s'y affirmer une stupeur face à l'étrangeté désormais acquise par le proche ou une blessure qui brutalement se rouvre, rendant les retrouvailles amères et douloureuses ${ }^{13}$.

Finalement, tous ces événements touchant à la densité expérientielle du retour nous ramènent avec insistance à l'expérience vive du détachement, à l'écart qui nécessairement se creuse entre des espaces existentiels, et notamment à l'impossible réciprocité qui mine le moment du départ. Car l'asymétrie de perception, faisant qu'un même phénomène se présente, pour celui qui part et celui qui reste, sous des angles inévitablement distincts, suppose des modalités actives et passives de vivre la séparation qui n'ont que peu de choses à voir entre elles et qui, probablement, ne convergeront jamais et pourront produire de l'équivoque aux retrouvailles. À la part d'ambiguïté que contient chaque départ répond un besoin de clarification dont la réponse consacre le moment des retrouvailles. Il reste à envisager comment les trois économies impliquées par le déplacement migratoire vont désormais être mises à contribution par ce besoin de clarification, parce qu'elles produisent notamment des articulations de sens et des noyaux d'intelligibilité susceptibles de raffermir les orientations existentielles fragilisées et de consolider la cohésion de vie menacée.

\section{Le problème de l'allègement de la dette contractée dans la rupture du départ}

Dans sa lecture de l'Essai sur le don, Paul Ricœur nous enseigne une chose cruciale : « la fascination exercée par l'énigme du retour conduit à négliger des traits remarquables de la pratique du don rencontrés en chemin », tels que risquer un premier don ou donner quelque chose de soi en donnant une simple chose matérielle (Ricœur,

13 Marcel Mauss invite, comme le remarque Bruno Karsenti, à percevoir une atmosphère diffuse de don capable de faire figurer un phénomène singulier au rang d'une expression ordinaire et structurante de la vie sociale (Karsenti, 1994 : 95-106). L'imprégnation émotionnelle qui tient à cette atmosphère ne concerne guère plus que la crainte de la sanction si les choses ne sont pas rendues (Mauss, 1993 : 212), associée à un sentiment pesant d'obligation compatible avec l'idée de rite (" on reçoit ainsi un don "sur le dos" » (Mauss, 1993 : 211). Dans sa conclusion générale, Marcel Mauss avoue toutefois avoir fait abstraction dans son étude de la « dimension esthétique des institutions » (portée par les chants, les parades ou les danses) et ainsi négliger les « émotions esthétiques ", celles qui touchent notamment à la joie de recevoir, au profit des " émotions de l'ordre du moral » (Mauss, 1993 : 274-275). Mais il ne parle pas non plus des retrouvailles, du fait que la circularité du don implique de se retrouver, de se voir à nouveau, d'insensiblement se rapprocher de l'autre. Or, l'importance et la difficulté des retrouvailles, et les migrants sont au cœur de cette question, tiennent précisément à une tension émotionnelle complexe qui se dégage puissamment du fait de revoir celui ou ceux dont on s'est senti ou dont on se sent encore proche. Elles sont ainsi bien souvent attirantes et effrayantes à la fois. 
2004 : 350). Il précise alors qu'il convient plutôt de mettre l'accent sur la seconde composante de la triade afin de pouvoir penser ensemble la totalité des phénomènes portant sur les intentions du don et le processus de reconnaissance qui est en jeu. Recevoir, dit Paul Ricœur en se penchant sur cette seconde composante, « devient alors la catégorie pivot, en ceci que la manière dont le don est accepté décide de la manière dont le donataire se sent obligé de rendre ». Afin de ne pas faire impérativement du retour le nœud de l'intrigue de la migration, un même effort de décentrement peut être effectué au niveau de la triade partir/rester/revenir.

En plaçant désormais l'attention sur la question du « rester », terme médian, voilà qu'on situe la qualité de l'expérience même de l'installation du migrant en regard de la manière dont est accepté son départ et de la façon dont il se sent obligé de revenir dans son pays d'origine. De ce point de vue, n'y a-t-il pas fatalement deux symptômes symétriques de l'échec d'une migration : d'un côté lorsque le retour est perçu comme une obligation impérative, ou de l'autre lorsqu'il est conçu comme un fait inenvisageable ${ }^{14}$. C'est aux alentours de ces deux pôles d'échec que pointe le soupçon de l'abandon. Que l'abandon soit motivé par un ressentiment et justifié par un dénigrement (du pays d'origine ou du pays d'accueil) n'empêche pas qu'il puisse laisser derrière lui, et notamment chez les proches, un profond sentiment d'ingratitude. La hantise de voir naître un tel soupçon, d'avoir à l'idée, par exemple, que les proches se sentent abandonnés après un départ, s'allie à la quête du rassurement qui s'atteste pleinement dans la préservation d'une gratitude réciproque. Lorsque cette gratitude se montre capable de résister aux séparations imposées par les départs et les retours, qui pourtant tranchent parfois violemment dans les liens établis, elle comble, en quelque sorte, l'inquiétude de l'abandon, et place la migration au rang d'un difficile parcours de reconnaissance.

La question épineuse des blessures de la migration ne peut donc pas être tenue à l'écart de celle des souffrances infligées, de manière largement non intentionnelle, par le migrant à ceux qu'il a laissés derrière lui, et qui donc l'ont vu partir vers un autre monde. La racine de ces lésions puise dans un faisceau d'événements à travers lequel s'est concrétisée l'intention d'une rupture ou la nécessité d'une séparation (projet de travail, exil forcé ou choisi, fuite, bannissement, regroupement familial, dispersion communautaire, etc.).

$14 \mathrm{Il}$ arrive fréquemment que dans les situations de déplacement subi, le migrant se retrouve finalement sur les deux pôles à la fois. Alors, un retour impossible peut l'obséder. Se dégager de cette impasse suppose de pouvoir soumettre son existence au fil conducteur des événements présents. Certains travaux sur l'exil forcé des réfugiés palestiniens montrent des choses de cet ordre. Sylvaine Bulle souligne que la puissante revendication du «droit au retour » des réfugiés s'accompagne d'un certain fatalisme sur les possibilités mêmes de ce retour (Bulle, 2009). Rappelant combien la nostalgie est pourtant l'expérience temporelle fondatrice de l'identité palestinienne exilée dans les camps, elle se montre particulièrement attentive à la manière dont le présent est paradoxalement réinvesti dans un foisonnement d'activités ordinaires touchant notamment au domaine du commerce. Ce présent figure en fait la « seule perspective vivable » car le retour finit par se fondre dans un « horizon inatteignable ». De même, le travail anthropologique effectué par Nicolas Puig dans un camp de réfugiés au Liban montre comment la nostalgie institutionnelle, orientée de longue date vers la mise en scène de la patrie perdue et la commémoration de la $N a k b a$, est réutilisée dans des projets musicaux actualisés et soumis à l'émergence de nouveaux styles (notamment le rap) et à des enjeux personnels visant l'émancipation des ordres collectifs et des relents les plus passéistes du combat politique (Puig, 2009).

REMI 2010 (26) 2 pp. 57-76 
C'est autour d'une première impression d'abandon que peut s'enrouler le « cercle vicieux de la réciprocité » (Ricœur, 2004 : 331) : au méfait de l'abandon succède un contre-méfait, s'élève le ressentiment, la jalousie, l'amertume ou la rancœur qui tous vont renforcer le pourrissement de la situation d'exil. Mais chacun des foyers de souffrance embrasés lors de la séparation doit ensuite être estimé à partir de la manière dont un lien est maintenu dans la distance, ce qui suppose de comprendre comment il est possible de le restaurer, qu'il se distende ou qu'il se tende au fil des épreuves de la migration. Au fond, une telle restauration ne peut s'appuyer que sur le pouvoir des migrants, et de ceux qui contribuent à irriguer ces épreuves, à enrayer le cercle vicieux de la réciprocité qui pourrit la situation d'exil et à continuer de nourrir une certaine reconnaissance mutuelle.

\section{POUR CONCLURE}

Que cette mutualité soit préservée en dépit des écarts subis, ceux que creusent l'éloignement des tonalités d'existence, le dérobement du côte-à-côte ou l'angoisse produite par l'absence, présuppose donc une capacité à produire et à tenir en estime une forme d'approbation de la séparation. Le ciment de la cohésion de vie du migrant n'est pas à chercher ailleurs. La confiance et l'assurance qui se dégagent de cette possible mutualité renforcent toutes les certitudes touchant au vers-où et au pour-quoi de l'orientation existentielle. Elles soutiennent une consistance personnelle face aux engagements comme aux désengagements que supposent les réorientations et les désorientations du phénomène migratoire. Elles facilitent le mouvement dans l'espace parcouru en lui donnant une assise et en lui offrant un sol porteur. Elles permettent que ce mouvement soit ouvert, que le corps déplacé s’y éprouve en sa cohésion, mais aussi en ses limites.

Et c'est précisément sur ces limites, là où le mouvement du déplacement du migrant connaît ses plus tangibles impasses et ses plus grandes faillibilités, que surgissent les enjeux politiques et éthiques de fond que sont ceux de la juste appartenance et de la bonne hospitalité (Stavo-Debauge, 2009). En mettant finalement au cœur de ces enjeux les questions de la réciprocité et de la mutualité, on a indiqué qu'ils concernaient indistinctement la communauté d'origine et la communauté d'accueil à tous les niveaux où le vivreensemble peut être défini et donc partout où des limites se définissent. Dans l'expérience de ces limites, non seulement le migrant prend conscience d'un partage des charges qui le tiennent lié aux communautés d'origine et d'accueil, mais il voit se jouer sous ses yeux, et par là même, l'épreuve de sa différence. Or cette épreuve appelle les dimensions normatives de la mise en ordre (de la place à tenir et à laisser) et de l'ajustement (de l'effort à entreprendre pour « bien » partir et « bien » venir). Ces dimensions de l'épreuve, qui touchent pour finir au gouvernement des hommes et des choses (oikonomia), demandent à être gérées aux plans de l'affectif et du pathique, de la morale et du symbolique. Sur chacun de ces plans se joue donc une économie différente. Ces plans sont particulièrement impliqués par le phénomène du déplacement que vit et subit le migrant car, au risque de menacer sa cohésion de vie, le déplacement impose des capacités de navigation et de conciliation bien particulières pour faire tenir ensemble des mondes distincts entre lesquels il faut bien trouver des lieux et des liens d'affinité, des points de passage et des lignes de continuité. Chaque économie produit certains bienfaits : limitation du regret, acquittement de la dette, affirmation d'une forme de réussite qui sont autant de manières de vivre une émigration 
autrement que sur le mode blessant de la réclusion, de l'égarement ou de la régression. C'est enfin à un élargissement des conceptions du phénomène migratoire qu'appelle notre enquête : appréhension d'un arc expérientiel comprenant les trois éléments interdépendants du partir, du rester et du revenir ; considération pour la dimension passive du vécu migratoire ; mise en jeu, enfin, d'une pluralité d'engagements tenant liés les migrants aux proches comme aux institutions qu'ils ont dû quitter et soulevant, par la même occasion, la complexité de l'enjeu politique qui s'y rattache.

\section{Références bibliographiques}

AYOUCH BODA Amina (1999) La nostalgie, exil, L'évolution psychiatrique, 64, 1999, pp. 271-280. BINSWANGER Ludwig (1987) Mélancolie et manie. Études phénoménologiques, Paris, PUF, $136 \mathrm{p}$.

BINSWANGER Ludwig (1998) Le problème de l'espace en psychopathologie, Toulouse, Presses universitaires du Mirail, $131 \mathrm{p}$.

BOLZINGER André (1999) La nostalgie selon Jaspers. Une thèse de médecine en 1909, L'évolution psychiatrique, 64, 1999, pp. 259-270.

BREVIGLIERI Marc (2001) L'étreinte de l'origine. Attachement, mémoire et nostalgie chez les enfants d'immigrés maghrébins, Confluences Méditerranée, 39, pp. 37-47.

BREVIGLIERI Marc (2002) L'horizon du ne plus habiter et l'absence du maintien de soi en public, in Daniel Céfaï et Isaac Joseph Éds., L'héritage du pragmatisme. Conflits d'urbanité et épreuves de civisme?, La Tour d'Aigues, Éditions de l'Aube, pp. 319-336.

BREVIGLIERI Marc et STAVO-DEBAUGE Joan (2004) Les identités fragiles. La « jeunesse » et l'« immigration » sous des regards sociologiques, in Catherine Cicchelli-Pugeault, Vincenzo Cicchelli et Tarik Ragi, Les jeunes. Liens, risques et engagements, Paris, PUF, pp. 159-176.

BULLE Sylvaine (2009) Domestiquer son environnement dans un territoire confiné : le camp de réfugiés de Shu'faat à Jérusalem-est, Genèses, 74, pp. 94-113.

DARDEL Eric (1990) L’homme et la terre. Nature de la réalité géographique, Paris, Éditions du CTHS, 1990, $201 \mathrm{p}$.

DUFOIX Stéphane (1998) Le retour des exilés, Hommes et Migrations, 1216, pp. 79-91.

FREY Jean-Marie (2004) "Le moi n'est pas maître dans sa propre maison ». Freud, Nantes, Éditions Pleins Feux, 47 p.

GIRAUD Vincent (2009) Le lieu et l'exil, Philosophie, 101, pp. 73-92.

HALBWACHS Maurice (1964), Esquisse d'une psychologie des classes sociales, Paris, Édition Marcel Rivière et Cie, $239 \mathrm{p}$.

HILY Marie-Antoinette et RINAUDO Christian (2003) Cosmopolitisme et altérité. Les nouveaux migrants dans l'économie informelle, Tsantsa, 8, pp. 48-57.

HOFER Johannes (1934) Medical dissertation on nostalgia, Bulletin of the History of Medecine, 2.

HOFER Johannes (1992), Dissertazione medica sulla nostalgia, traduit in Prete, A. (a cura di), Nostalgia. Storia di un sentimento, Rafaello Cortina Editore, Milano, pp. 45-59.

JANKÉLÉVITCH Vladimir (1974) L'irréversible et la nostalgie, Paris, Flammarion, 319 p.

KARSENTI Bruno (1994) Marcel Mauss. Le fait social total, Paris, PUF, 128 p.

LAFFORT Bruno (2005) Le mythe du retour, Hommes et Migrations, 1253, pp. 105-116.

MAUSS Marcel (1993) Sociologie et anthropologie, Paris, Quadrige/PUF, 482 p.

PARK Robert Erza (1984) La ville. Propositions de recherche sur le comportement humain en milieu urbain, in Yves Grafmeyer et Isaac Joseph, L'école de Chicago. Naissance de l'écologie urbaine, Paris, Éditions Aubier, pp. 83-130. 
POTOT Swanie (2007) Vivre à l'Est, travailler à l'Ouest : les routes roumaines de l'Europe, Paris, L'Harmattan, $227 \mathrm{p}$.

PUIG Nicolas (2009) Exils décalés. Les registres de la nostalgie dans les musiques palestiniennes au Liban, Revue Européenne des Migrations Internationales, 25 (2), pp. 83-100.

RAUCHS Paul (1999) La nostalgie ou le malentendu du retour, L'évolution psychiatrique, 64, 1999, pp. 281-288.

RICOEUR Paul (1991) De la philosophie au politique, in Paul Ricœur Lectures 1, Paris, Seuil, pp. 15-19.

RICOEUR Paul (2000) La mémoire, l'histoire, l'oubli, Paris, Éditions du Seuil, 676 p.

RICOEUR Paul (2004) Parcours de la reconnaissance. Trois études, Paris, Stock, 387 p.

SARTHOU-LAJUS Nathalie (1997) L'éthique de la dette, Paris, PUF/Questions.

SAYAD Abdelmalek (1999) La double absence. Des illusions aux souffrances de l'immigré, Paris, Seuil, $439 \mathrm{p}$.

SERFATY-GARZON Perla (2006) Enfin chez soi ? Récits féminins de vie et de migration, Montréal, Bayard Canada Livres, $188 \mathrm{p}$.

SIMMEL Georg (2002), La philosophie de l'aventure. Essais, Paris, L'arche Éditeur, 123 p.

STAVO-DEBAUGE Joan (2009) Venir à la communauté. Une sociologie de l'hospitalité et de l'appartenance, thèse de doctorat, EHESS, $965 \mathrm{p}$.

THÉVENOT Laurent (2006) L'action au pluriel. Sociologie des régimes d'engagement, Paris, Éditions La Découverte, $311 \mathrm{p}$. 


\title{
De la cohésion de vie du migrant : déplacement migratoire et orientation existentielle
}

\author{
Marc BREVIGLIERI
}

Le déplacement migratoire recèle schématiquement deux tendances prêtes à se dissocier : l'une qui cherche à déplacer intégralement le lieu d'origine, à l' « emmener » avec soi, et la nostalgie relève de cette tendance, et l'autre qui ignore ou abdique devant un tel effort, semblant fuir ce qui touche au passé, répondre d'un besoin de renaître ou de la tentation de l'oubli. Chacune de ces tendances place au cœur de l'espace existentiel le problème de l'orientation depuis lequel migrer pose les questions du vers-où et du pour-quoi de l'existence. Ce texte entend établir les enjeux du déplacement migratoire au niveau de la cohésion de vie du migrant. Ce niveau permet de revenir sur la grande diversité des blessures de la migration (mélancolie et sentiment du manque, culpabilité et remords, frustration et humiliation), de comprendre comment elles s'ouvrent, se referment et peuvent être dépassées dans une gestion qui, loin de ne renvoyer qu'au soi du migrant, met en jeu simultanément les communautés d'origine et d'accueil.

\section{The Life-Cohesion of the Migrant: Migration and Existential Orientation}

\author{
Marc BREVIGLIERI
}

Migration generates two distinct dispositions. The first one involves a nostalgic desire to take with oneself the place of origin in its entirety. The other disposition, seeming to escape all that pertains to the past, answers to the imperative of being reborn and to the temptation of forgetting. Each disposition places at the heart of the existential space a problematic of orientation, from which the migrant formulates the questions of the toward-where and for-what of existence. This article attempts to situate the stakes of migration at the level of the life-cohesion of the migrant. This level enables us to reconsider the full range of the wounds inflicted by migration (melancholia and loss, guilt and remorse, frustration and humiliation). It also allows us understand how these wounds open, heal up, and possibly put behind through a management which, far from only involving the self of the migrant, implicates simultaneously both the community of origin and the community of reception.

\section{Sobre la cohesión de vida del migrante: desplazamiento migratorio y orientación existencial}

\section{Marc BREVIGLIERI}

El desplazamiento migratorio supone esquemáticamente dos tendencias prestas a disociarse: una que intenta desplazar integralmente el lugar de origen y llevarlo consigo, la nostalgia encontrando allí un soporte; la otra ignora o abdica ante tal esfuerzo, como si huyera lo que tiene que ver con el pasado, respondiendo así a la tentación del olvido o a la necesidad de renacer. Dichas tendencias centralizan el problema de la orientación que el migrar da al "hacia donde" y el "porque" de la existencia. Este texto reflexiona sobre los objetivos del desplazamiento migratorio en relación a la cohesión de vida del migrante. Esta relación permite discurrir sobre la gran diversidad de heridas de la migración (melancolía y falta, culpabilidad y remordimientos, frustración y humillación), comprendiendo como estas se abren, se cierran y pueden ser sobrepasadas por una gestión que pone en juego simultáneamente las comunidades de origen y de llegada, en lugar de restringirla solamente al propio migrante. 